\title{
Meet the human metabolome
}

Imagine that at a routine medical check-up your doctor takes a urine sample, then reports a few days later that your risk of type 2 diabetes is normal, but there are hints that your arteries are furring up.

A similar scenario has been promised for the past 20 years by those working in genomics and proteomics, but has not yet materialized. Now, however, an increasing number of researchers are claiming that metabolomics - the study of all the body's metabolites - will finally come up with the goods.

Supporters of this burgeoning branch of molecular medicine are gung-ho about their chances of success. "In retrospect, we wonder why we spent millions on the genome," says Bruce German, who studies lipid metabolism at the University of California, Davis. With the knowledge we have today, he reckons, scientists should have gone straight for the metabolome. But can it deliver?

Metabolomics is the study of the raw materials and products of the body's biochemical reactions, molecules that are smaller than most proteins, DNA and other macromolecules. The aim is to be able to take urine, blood or some other body fluid, scan it in a machine and find a profile of tens or hundreds of chemicals that can predict whether an individual is on the road to a disease, say, or likely to experience side-effects from a particular drug.

Researchers are already trying to flag impending disease by measuring levels of gene expression or proteins, but supporters of metabolomics say they should be able to do it better. Small changes in the activity of a gene or protein (which may have an unknown impact on the workings of a cell) often create a much larger change in metabolite levels. The approach has already proved its worth: cholesterol and glucose have long been chemical canaries for heart disease and diabetes.

\section{Data fingerprinting}

But realizing this vision isn't straightforward. One of the first tasks is to create a catalogue of compounds in the human body, and this is proving hard to define. David Wishart at the University of Alberta, Edmonton, and his colleagues have taken an initial step forward by producing something they rather grandly call the first draft of the human metabolome ${ }^{1}$. They searched the published literature for known human metabolites, and have collected around 2,500 of them into a public database (www.hmdb.ca) along with other information such as known links to disease. The researchers also used nuclear magnetic resonance and mass spectroscopy to produce characteristic 'fingerprints' for more than 400 compounds, and have added these to the database.

It's the most comprehensive collection of metabolite data to be made publicly available. But others in the field point out that Wishart's catalogue is far from complete because the number and nature of compounds in the human metabolome will vary depending on which body fluid is looked at and the method used for the analysis. There is also no clear division between compounds produced by the human body, those produced by our gut bacteria and fleeting products generated by food or drugs swallowed that day.

"The notion that this is a first draft of the human metabolome is nonsense," says Jeremy Nicholson of Imperial College London, one of the pioneers of the field. "I agree that it covers a helluva lot of important metabolites, but it's a very arbitrary guess at what might be useful and what might not."

An added complication is that one person's profile of metabolites is likely to be dramatically different from another's, and each may fluctuate markedly depending on the time of day, what they last ate and other aspects of their lifestyle. To get a handle on this variation, Nicholson has studied tens of thousands of urine samples from many ethnic groups around the world and found that each group is remarkably different. A separate study showed that the metabolic profile of meat eaters is very different from that of vegetarians ${ }^{2}$. This means that a person's metabolome might need to be measured many times during their lives in order to be able to pick out changes that might

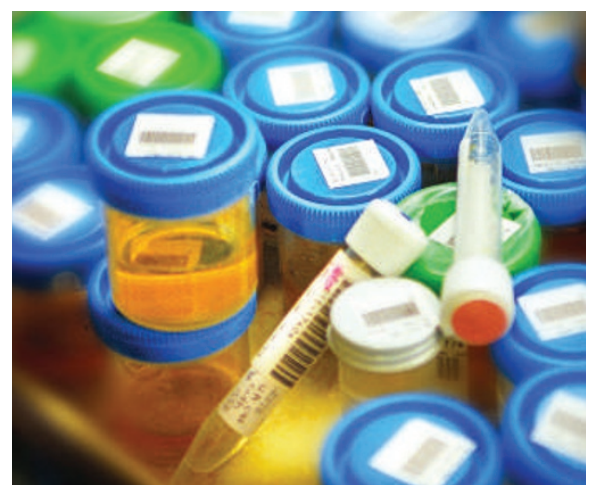

Hopes are high that in future a urine sample might reveal our health profile from our metabolites. signal disease. Also, any one metabolite will have to be assessed relative to the pattern of many others.

Whatever the total metabolite tally, researchers will have to prove that particular concentrations and combinations can reveal something about drugs or disease. Preliminary studies suggest that this can be done. Last year, for example, Nicholson and his colleagues showed that a fingerprint of the metabolites in urine could predict which rats would suffer liver damage from the drug paracetamol ${ }^{3}$. He says he has now shown the same in humans, and is preparing the study for publication. A team led by Douglas Kell at the University of Manchester, UK, has developed a computer model based on metabolite profiles in blood plasma that can identify pregnant women with the dangerous condition called pre-eclampsia ${ }^{4}$.

\section{Lab on a chip}

But just as with genomics and proteomics, finding profiles that reliably predict the onset of disease will be a major undertaking, because it will typically require sampling regular profiles from many thousands of people and then following them for years to see which ones develop a particular condition. Researchers are hopeful that biobanks - large collections of people's biological samples and medical records - will in future supply this information, but such studies could take decades. They will also have to learn from those working on gene-expression or proteomic profiles, who have sometimes struggled to show that a test that works in one group also works in another, or that the changes they see are actually involved in a disease. "In my opinion we should know why this metabolite is going up or down," says William Bigbee, an expert in biomarkers and proteomics at the University of Pittsburgh Cancer Institute, Philadelphia.

In the long run, the best way to predict an individual's disease risk is likely to come from understanding the biology behind each disease - and that will come from a combination of genomics, proteomics and metabolomics. "I don't want to buy six machines," says Ben van Ommen of the Netherlands Organization for Applied Scientific Research in Zeist. "I want a lab on a chip that measures metabolites, proteins and gene expression."

Helen Pearson

4. Kenny, L. C. et al. Metabolomics 1, 227-234 (2005). 